\title{
Sensibilidad, comportamiento de base segura y desarrollo sociocognitivo en centros de educación inicial en México*
}

\author{
Sensitivity, Secure Base Behavior and Socio-Cognitive \\ Development in Mexican Childcare Settings
}

Recibido: 12 de febrero de 2015 | Revisado: 30 de junio de 2015 | Aceptado: 10 de julio de 2015

FERNANDO SALINAS-QUIROZ **

Universidad Pedagógica Nacional, México

doi:10.11144/Javeriana.upsy14-3.scbs

Para citar este artículo: Salinas-Quiroz, F. (2015). Sensibilidad, comportamiento de base segura y desarrollo sociocognitivo en centros de educación inicial en México. Universitas Psychologica, 14(3), 1017-1028. http://dx.doi.org/10.11144/Javeriana. upsy14-3.scbs

Este artículo se deriva de la investigación denominada "Educación Inicial de Base Segura: Estudio observacional de los procesos internos de la educadora, de la interacción y del desarrollo sociocognitivo infantil" presentada por el autor para optar al título de Doctor en Psicología, Universidad Nacional Autónoma de México (UNAM), 2014. Al Programa de Maestría y Doctorado en Psicología de la UNAM y al Consejo Nacional de Ciencia y Tecnología por el financiamiento del proyecto.

** Profesor-Investigador de la Universidad Pedagógica Nacional. Doctor en Psicología, UNAM. Miembro del Sistema Nacional de Investigadores, del Programa de para el Desarrollo Profesional Docente y de la Red Iberoamericana de Apego. Correo electrónico: fsalinas@upn.mx

\section{RES UMEN}

La calidad del cuidado una variable es determinante en el desarrollo infantil temprano: en Centros de Educación Inicial (CEI), las interacciones Cuidador Secundario Profesional (CSP)-infante constituyen el núcleo de dicho proceso. El propósito de esta investigación fue evaluar la relación entre la sensibilidad de las CSP y el comportamiento de base segura de los infantes con su desarrollo sociocognitivo. Participaron 34 díadas CSP-infante de CEI del Instituto Mexicano del Seguro Social. Se utilizó el MBQS, el AQS y la EECSC. Las CSP tuvieron niveles de sensibilidad adecuados $(M=0.59)$, pero los infantes no construyeron vínculos de apego con ellas $(M=0.26)$ debido al corto tiempo de interacción. Tanto la respuesta sensible activaanimada de las CSP $(r=0.51)$ como el comportamiento de base segura de los infantes $(r=0.4)$ se asociaron con su desarrollo sociocognitivo. Se sugiere que las CSP permanezcan con el mismo grupo de infantes durante toda su estancia en el CEI.

Palabras clave

Teoría del apego; sensibilidad; comportamiento de base segura; desarrollo infantil temprano; calidad educativa

\section{A B S T R A C T}

Quality of care is a potentially important determinant of child outcomes. Professional Secondary Caregiver (PSC)-child interactions constitute the core of process quality. The purpose of the study was to examine the association between the PSC's sensitivity, child security -as assessed with the scores derived from Q-Sort descriptions (MBQS and AQS) - and socio-cognitive development (as derived from an assessment) in an accidental sample of 34 dyads trough a cross-sectional study. PSC were highly sensitive $(\mathrm{M}=.59)$ but children had less than 4 months interacting with them and showed it was insufficient time to establish a secure base relationship. Adequate and appropriate responses from the nonmaternal caregiver to communication and signs the infant is making relate to the child's socio-cognitive development. More stable and long term PSC-child relationships would lead to better outcomes in child development, therefore, allowing the PSC to spend more time with the same group of children would be ideal.

Keywords

Attachment Theory; Sensitivity; Secure Base Behavior; Child Development; Educational Quality. 
A nivel mundial, en la última década, el número de infantes que asisten a Centros de Educación Inicial (CEI), popularmente conocidos como guarderías, estancias infantiles o salas cuna, aumentó cerca de un $40 \%$ (Organización de las Naciones Unidas para la Educación, la Ciencia y la Cultura [UNESCO], 2012). Investigaciones sobre su calidad documentan que la asistencia a CEI de baja calidad es un factor de riesgo (p. ej., Friedman \& Boyle, 2008), mientras que su alta calidad es un factor protector en el desarrollo infantil temprano (p. ej., Gunnar, Tagle, \& Herrera, 2009; Vandell et al., 2010; Vermeer \& Bakermans-Kranenburg, 2008).

Las interacciones Cuidador Secundario Profesional (CSP)-infante constituyen el núcleo de dicho proceso (Cárcamo, Vermeer, De la Harpe, van der Veer, \& van Ijzendoorn, 2014). La teoría del apego resulta un excelente marco de referencia para estudiar la sensibilidad de los CSP y su vinculación con los infantes, generando posibles indicadores para evaluar la calidad educativa (Salinas-Quiroz et al., 2015). Un cuidador sensible centra su atención en las necesidades físicas y emocionales del infante y es capaz de ver las cosas desde su punto de vista. Asimismo, sincroniza las actividades del niño o la niña con las propias, negocia ante conflictos y se ajusta a sus estados emocionales, momento evolutivo y particularidades (p. ej., Carbonell, Posada, Plata, \& Méndez, 2005). La organización de las conductas de base segura tienen como fin el mantenimiento de la proximidad entre el individuo y una o varias personas afectivamente cercanas a él (Salinas-Quiroz, 2013).

Investigaciones recientes han reportado que la relación de base segura infante-CSP permite que el niño transforme al CSP en un recurso para explorar el aula (Oren, 2006) y pueda enfocar toda su atención y energía en el aprendizaje (p. ej., Howes $\&$ Richie, 2002). Una base segura protectora ofrece una variedad de oportunidades de aprendizaje bajo supervisión: interacciones exploratorias con objetos y personas que alcanzan sus niveles óptimos con el apoyo de la figura de apego (Salinas-Quiroz et al., 2015). Las relaciones de base segura infante-CSP predicen, tanto en Educación Inicial (EI) como en niveles superiores: a) el ajuste conductual de los infantes; b) su aceptación social; c) sus actitudes escolares y d) su compromiso y desempeño académico (p. ej., Baker, 2006; Kienbaum, 2001; Magnuson, Ryhm, \& Waldfogel, 2004; Pianta \& Stuhlman, 2004; Silver, Measelle, Armstrong, \& Essex, 2005). Otros estudios han encontrado que la calidad de la relación infante-CSP predice mejores niveles de competencia social (p. ej., Maldonado \& Carrillo, 2006; Peisner-Feinberg et al., 2001; Pianta \& Stuhlman, 2004) y disminuye problemas conductuales (p. ej., Mashburn, Pianta, Hamre, \& Downer, 2008).

En México, los derechos de la infancia han sido incorporados a la Ley General de Educación (2011), por lo que la Secretaría de Educación Pública reconoce como un derecho inalienable de la infancia el derecho a la EI. El análisis del gasto realizado por el Banco Interamericano del Desarrollo en 2013 revela inequidades en los servicios de EI en el país: la cantidad destinada a las hijas y los hijos de derechohabientes de instituciones de seguridad social (p. ej., Instituto Mexicano del Seguro Social [IMSS] e Instituto de Seguridad y Servicios Sociales de los Trabajadores del Estado [ISSSTE]) equivale a 2.5 veces más de lo que se asigna para la población no derechohabiente, a pesar de que la cantidad de infantes es menor (Myers, Martínez, Delgado, Fernández, \& Martínez, 2013), por lo que se espera que la calidad del cuidado en los CEI del IMSS sea superior. La Ley General de Prestación de Servicios para la Atención, Cuidado y Desarrollo Integral Infantil (2011) reconoce que los entes que prestan servicio de cuidado infantil y EI deben llevarlo a cabo conforme a estándares de calidad, donde lo prioritario sea la adecuada atención de las niñas y los niños, basados en el principio constitucional que tutela su desarrollo y el cumplimiento de sus derechos, es decir, en el interés superior de la infancia.

La investigación existente sobre los efectos que tiene la asistencia a CEI en el desarrollo infantil temprano se ha realizado principalmente en contextos anglosajones. El mayor estudio longitudinal realizado no encontró interacciones entre el temperamento y las características del CEI, sin embargo, no consideró en su diseño la evaluación de la interacción infante-CSP. Autores como Rois- 
man y Fraley (2008) sugieren que el modelo más adecuado para explicar la correlación sensibilidadseguridad es el ambiental no el genético, por lo que la calidad del cuidado se convierte en una variable importante. Una manera de aproximarse a dichas interacciones es a través de la evaluación de los vínculos de apego y la sensibilidad del CSP.

Dentro del contexto latinoamericano, específicamente en Chile, se ha evaluado la interacción infante-CSP con el Índice Experimental de Relación Niño-Adulto (Care-Index) (Crittenden, 2005) y con la Escala de Sensibilidad del Adulto (ESA) (Santelices et al., 2012). Ambos instrumentos requieren de la videograbación del juego libre entre el infante y el cuidador durante 3 a 5 minutos y sus indicadores evalúan aspectos afectivos y cognitivos de la respuesta sensible (Santelices \& Pérez, 2013; Santelices et al., 2012). El equipo de investigación chileno encontró que con el paso del tiempo la respuesta sensible de los CSP en CEI se mantiene; el personal educativo disminuye su estilo controlador en la interacción (Santelices \& Pérez, 2013) y la cooperatividad aumenta de manera significativa (Santelices, Greve, \& Pereira, 2015). En 2015, evaluaron las variables predictores de la sensibilidad materna en una muestra de 104 díadas madre-hijo de un año de edad que asistían a CEI y encontraron que a mayores niveles educativos u ocupacionales hay una mayor probabilidad de que la madre sea más sensible con su hijo, empero, respecto a los infantes, no encontraron correlaciones significativas entre el sexo, edad, edad de ingreso, horas semanales en el CEI, tipo de hijo, ni el lugar que ocupa entre hermanos (Santelices, Farkas et al., 2015). Respecto a la comparación de la sensibilidad de las madres chilenas con las CSP, Farkas y colaboradoras (2015) compararon la sensibilidad de madres y CSP por medio de la ESA (Santelices et al., 2012). En el caso de las madres, encontraron correlaciones estadísticamente significativas entre mayores niveles de sensibilidad y más edad, nivel educativo y nivel socioeconómico; las CSP con mayores niveles de sensibilidad también tuvieron niveles educativos más elevados y menor tiempo a cargo del infante evaluado (Farkas et al., 2015).

La evaluación de la sensibilidad y de las relaciones de base segura infante-CSP, así como el desarrollo sociocognitivo de niñas y niños en CEI de instituciones de seguridad social mexicanas, no solo es pertinente para fines investigativos de divulgación, con el respectivo aporte a la teoría del apego respecto a la multiplicidad de cuidadores y asistencia a CEI en contextos latinoamericanos, sino que documenta la calidad del cuidado y atención educativa que reciben los infantes con miras a mejorarla en cumplimiento de la convención de los derechos de las niñas y los niños (Fondo Internacional de Emergencia de las Naciones Unidas para la Infancia [UNICEF], 1989). La hipótesis de partida del presente estudio indica que la sensibilidad de las CSP y el comportamiento de base segura de los infantes se asociarán con su desarrollo sociocognitivo.

\section{Método}

\section{Vinculación institucional}

Se sostuvieron tres distintas reuniones con la coordinación del Servicio de Guarderías para el Desarrollo Integral Infantil del IMSS durante el primer semestre del 2013 para presentar el proyecto de investigación, solicitar su participación y seleccionar CEI de buena o excelente calidad. Una vez concluidas las negociaciones, las autoridades propusieron que participaran en el proyecto CEI de prestación indirecta únicamente (subrogadas), por lo que se seleccionaron cuatro que probablemente tendrían dichos niveles de calidad, al sur de la Ciudad de México para facilitar el transporte del equipo de investigación. En el mes de agosto del 2013, se realizó la presentación formal de la investigación al equipo directivo de los cuatro CEI participantes (CEI1, CEI2, CEI3 y CEI4) para proceder con la evaluación de la calidad y, en caso de que cumplieran con los estándares preestablecidos, realizar un cronograma de tiempos y detallar pormenores para comenzar la investigación. 


\section{Criterios de inclusión de los CEI}

La selección de los CEI se hizo con base en la Escala de Evaluación de Calidad de Centros de Educación Inicial ([EECCEI, v.4.0]; UPN, 2011); participaron en la investigación aquellos que cumplieron con niveles de calidad buenos o excelentes según el instrumento ( $\geq 80 \%$ ). La EECCEI evalúa los indicadores de la calidad del centro y de la sala. Los niveles de calidad son: 1) Inadecuado $\leq 20 \%$; 2) Incipiente $\leq 40 \%$; 3) Básico $\leq 60 \%$; 4) Bueno $\leq 80 \%$ y 5) Excelente $=100 \%$. Para aplicar la escala, se entrevistó a la directora de cada uno de los cuatro CEI; posteriormente, se procedió con la observación directa de las condiciones físicas de la infraestructura y de los recursos de los CEI. Se calificaron las cuatro dimensiones de la escala así: 1. Instalaciones y Recursos Materiales; 2. Gestión Escolar; 3. Proceso Educativo y 4. Relación con las Familias. Respecto a las Instalaciones y Recursos Materiales, el CEI1 cumplió con un 95 \%; el CEI2, $100 \%$; el CEI3 y el CEI4, 80 \%. En cuanto a la Gestión Escolar, los CEI1, CEI2 y CEI4 obtuvieron el $100 \%$ y el CEI3, el $92 \%$. Sobre el Proceso Educativo, los CEI1, CEI2 y CEI4 cumplieron al $100 \%$ y el CEI3, con el $80 \%$. Respecto a la Relación con las Familias, solo el CEI2 obtuvo el $100 \%$, mientras que el CEI3 y el CEI $4,93.3 \%$ y el CEI1 el $86.7 \%$.

\section{Criterios de inclusión de las díadas}

En el caso del CSP, fue requisito ser mujer, contar con una carrera técnica de asistente educativo o puericulturista o tener experiencia en sala maternal, MA: 18 a 24 meses; B1: 25 a 30 meses; o B2: 31 a 36 meses, según la nomenclatura del IMSS. En el caso de los infantes, tener de 18 a 36 meses de edad al momento de la evaluación y estar sanos, según reportes de los distintos servicios del CEI (salud, vacunación, higiene, nutrición y seguimiento de peso y talla, psicología y trabajo social).

\section{Participantes}

De las 34 díadas CSP-infante: $50 \%$ fue niñas ( $n=$ 17) y $50 \%$, niños $(n=17)$ de 20 a 36 meses de edad
$(\mathrm{M}=27.94 ; \mathrm{DE}=5.49)$. Las CSP tenían entre 18 y 42 años de edad $(M=29.26 ; D E=6.30)$, todas contaban con al menos una carrera técnica y entre 1-240 meses de experiencia profesional $(M=67.41$; $D E=60.88)$. El tiempo de interacción de las díadas fluctuó entre 4 y 8 meses $(M=3.69: D E=1.76)$.

\section{Instrumentos}

\section{Sensibilidad de la CSP}

Se utilizó el Q-Sort del Comportamiento del Cuidador Secundario Profesional ([QSCCSP]; Salinas-Quiroz, 2014), adaptación del Q-Sort del Comportamiento Materno ([QSCM; Pederson \& Moran, 1995). Se revisó la traducción al castellano de Posada y equipo (Posada, Jacobs, Carbonell et al., 1999; Posada, Jacobs, Richmond, 2002) y la de Juárez-Hernández (2004a); se adaptó el lenguaje al contexto mexicano y se modificó el cuidador, madre, por el de este estudio, CSP. Para esta adaptación, se utilizó una validación por jueces (SalinasQuiroz, Morales-Carmona, Cruz-Martínez, Posada, \& Carbonell, 2014). El QSCM se asocia con la metodología cuantitativa, ya que asigna puntajes, además combina las fuerzas y metodología del investigador cualitativo, pues permite hacer un estudio sistemático de la subjetividad (Salinas-Quiroz \& Posada, 2015).

Posada, Waters, Crowell y Lay (1995), con base en criterios teóricos, construyeron siete escalas que han sido utilizadas en diversas investigaciones con madres en el medio latinoamericano (p. ej., Posada et al., 2002), son estas: 1) Respuesta sensible $(n=12, \alpha=0.92)$; 2) Accesibilidad ( $n$ $=9, \alpha=0.88)$; 3) Aceptación del infante $(n=6$, $\alpha=0.83)$; 4) Interferencia $(n=3, \alpha=0.76)$; 5) Activa-Animada ( $n=8, \alpha=0.89) ; 6)$; Crea un ambiente interesante $(n=5, \alpha=0.8)$ y 7) Preocupación por la apariencia física $(n=3,0.73)$. En 2014, Salinas-Quiroz y colaboradores realizaron una aproximación psicométrica al uso de los QSorts en contextos de EI, mediante un análisis factorial exploratorio en el que se conservaron 49 reactivos de los 90 originales que convergieron en 16 interacciones en 3 factores que explicaron 
SENSIBILIDAD, COMPORTAMIENTO DE BASE SEGURA y DESARROllo

sociocognitivo en Centros de educación inicial en MÉxico

el $45.35 \%$ de la varianza: 1) Comportamiento sensible ( $n=28, \alpha=0.92)$; 2) Rigidez y lineamientos institucionales $(n=8, \alpha=0.72)$ y 3$)$ Promoción del aprendizaje $(n=13, \alpha=0.87)$ (Salinas-Quiroz et al., 2014).

Para la presente investigación, se empleó el puntaje global del Q-Sort, además de los puntajes de cada una de las escalas de Posada et al. (1995). El puntaje global del QSCCSP se obtiene mediante una correlación entre la cuidadora prototípicamente sensible y la CSP evaluada (-1 a 1$)$.

\section{Comportamiento de base segura}

Se utilizó el Q-Sort del Apego, QSA versión 3.0 (Waters, 1995) el cual cuenta con 90 reactivos y ha sido previamente traducido al castellano por Posada et al. en Colombia (Posada et al., 1999; Posada et al., 2002) y Juárez-Hernández en México (2004b). A partir de sus traducciones, se diseñó una versión con lenguaje adaptado al contexto mexicano y modificaciones en el tipo de cuidador, adaptado para CSP. Para esta adaptación también se utilizó una validación por jueces (SalinasQuiroz et al., 2014).

Posada, Jacobs, Carbonell et al. (1999) y Posada, Jacobs, Richmond et al. (2002) construyeron cuatro escalas con base en criterios teóricos, a saber: 1) Interacciones cálidas $(n=17, \alpha=0.91) ; 2)$ Proximidad ( $n=13, \alpha=0.77) ; 3)$ Contacto físico $(n=7, \alpha=0.8)$ y 4$)$ Interacción con otros adultos $(n=13, \alpha=0.81)$. La puntuación promedio de los reactivos que componen cada escala constituye el nivel de dicha dimensión en la conducta de base segura de los infantes. Salinas-Quiroz et al. realizaron un análisis factorial exploratorio en el que se conservaron 47 reactivos de los 90 originales que convergieron en 48 interacciones en 6 factores que explicaron el $52.99 \%$ de la varianza: 1) Interacciones armoniosas y búsqueda de la proximidad con la CSP $(n=15, \alpha=0.87)$

2) Disposición y respuestas emocionales positivas a extraños $(n=7, \alpha=0.9)$; 3) Inconsistencia en el vínculo con la CSP $(n=11, \alpha=0.63) ; 4)$ Contacto físico con la CSP $(n=4, \alpha=0.57) ; 5)$ Necesidad de atención de la CSP $(n=6, \alpha=$
0.63) y 6) Estados emocionales positivos ( $n=4$, $\alpha=0.65)$ (Salinas-Quiroz et al., 2014).

Se empleó el puntaje global del Q-Sort, además de los puntajes de cada una de las escalas de Posada y cols. (Posada et al., 1999; Posada et al., 2002). El puntaje global del QSA se obtiene mediante una correlación entre el infante prototípicamente seguro y el infante evaluado (-1 a 1$)$.

\section{Desarrollo sociocognitivo}

Fue medido con la Escala Evaluación de Capacidades Sociales y Cognitivas ([EECSC]; JuárezHernández et al., 2013). Dicha escala evalúa los indicadores de desarrollo de las capacidades sociales y cognitivas de infantes de uno y dos años de edad. Se emplearon dos versiones: una para infantes de 12 a 23 meses; otra para los de 24 a 35 meses. La primera incluye dos categorías: 1) Identidad personal y social y 2) Regulación; la segunda, tres categorías: 1) Razonamiento, 2) Pensamiento deductivo emergente y 3) Identidad personal y social. Mientras que la escala de un año comprende un total de siete reactivos, la de dos años tiene trece. Cada capacidad cognitiva o social es medida en sus dos dimensiones: producto (respuesta) y proceso (nivel de dominio de la respuesta). Las escalas son de tipo Likert y tienen la siguiente estructura: i. Indicador que se evalúa; ii. Situación de evaluación; iii. Consigna o pregunta y iv. Escala con cinco valores numéricos (1-5) para clasificar cinco posibles tipos de respuesta cualitativamente distintas: (1) Sin-respuesta, (2) Respuesta-no-pertinente, (3) Pertinente-incipiente, (4) Pertinente-dominio y (5) Pertinente-sobresaliente. Respecto a su confiabilidad, se reportó un alpha de Cronbach de $\alpha=0.73$ que explica un $46.32 \%$ de la varianza para la escala de un año de edad y $\alpha=0.83$ para la escala de dos años, explicando el $52.33 \%$ de la varianza total (Juárez-Hernández et al., 2013). Los reactivos se calificaron en una escala de cinco puntos con base en la complejidad de la respuesta de los infantes. Se utilizaron los subtotales de cada categoría, así como el total de toda la escala. 


\section{Consideraciones éticas}

Se le solicitó a las directoras de los 4 CEI seleccionados una breve reunión con las CSP para leerles la carta de consentimiento informado (Sociedad Mexicana de Psicología [SMP], 2007), explicando las características del estudio y enfatizando la confidencialidad de la información. Como agradecimiento a su participación, se ofreció una reunión de devolución de resultados junto a la directiva de los 4 centros. En el caso de los infantes, a la hora de su ingreso a los CEI, las directoras abrieron la convocatoria a los padres de familia a participar, dándoles la carta de consentimiento informado (SMP. 2007), la cual explica las características del estudio, enfatiza en el anonimato y confidencialidad de los datos, y especifica que no les sería devuelto ningún tipo de resultado, esto como consecuencia ante la imposibilidad real de ofrecer una devolución a los padres, además de que se asumió que no se encontrarían anomalías en el desarrollo de los infantes, ya que fueron requisitos de inclusión estar sanos según reportes del CEI. La EECSC propone situaciones problemáticas a los infantes para que las resuelvan y tiene como prerrequisitos: a) contar con la presencia y el acompañamiento de un cuidador conocido, y b) que el infante se encuentre dispuesto a participar. Si alguno de estos dos criterios no se cumplía, no se procedió con la evaluación de su desarrollo social y cognitivo.

\section{Procedimiento}

Aquellos infantes que contaban con los consentimientos firmados fueron aleatorizados mediante una tómbola para ser asignados a una CSP en particular. La evaluación de cada díada duró una semana y al menos cuatro observadores estuvieron involucrados: un par de observadores evaluó la sensibilidad de la CSP en dos momentos distintos (una visita de hora y media el miércoles a la hora del desayuno y otra de misma duración el jueves en la sala); otro par de observadores independientes evaluó el comportamiento de base segura del infante con la CSP en dos momentos distintos (dos visitas de hora y media cada una los días lunes y martes; la del lunes durante el desayuno y la del martes en la sala o recreo). Los jueves dos asistentes de investigación evaluaron el desarrollo sociocognitivo de los infantes con la EECSC.

\section{Resultados}

En cuanto a la sensibilidad global de las CSP, se encontró una media de $0.59(D E=0.13)$, lo cual muestra niveles de sensibilidad adecuados. Respecto a las escalas, existen diferencias entre las medias de las CSP participantes y los promedios de las escalas, lo que permite dilucidar diferencias entre las características de la sensibilidad materna y los rasgos de las CSP estudiadas. En Respuesta Sensible, las media para CSP fue de 6.55, mientras que la media de la escala con madres es de 5.04; en cuanto a la Accesibilidad, las CSP tuvieron una media de 6.02 y las madres $M=5.17$; Aceptación del infante, CSP $\mathbf{M}=6.38$ versus una media de 4.78 en mamás; Interferencia, $\operatorname{CSP} M=7.60$, media de la escala con madres 1.56; Activa-animada, $M=6.23$ con CSP y $\mathrm{M}=5.72$ con mamás; Crea un ambiente interesante, CSP $M=7.59$, media de madres igual a 5.9 y Preocupación por la apariencia física, CSP M = 4 , mientras que la media de la escala con mamás es de 3.89 .

El comportamiento de base segura de los infantes con la CSP tuvo una media de 0.26, DE $=0.16$, lo que indica que no han construido una relación de base segura con su CSP. Correspondiente a las escalas del Q-Sort del Apego propuestas Posada et al. (Posada et al., 1999; Posada et al., 2002), también existen diferencias entre las medias de los participantes y los puntajes promedios de las escalas, lo que ilustra la variabilidad de la organización de las conductas de base segura con diferentes cuidadores. En Interacciones cálidas, los infantes participantes tuvieron una media de 7 , mientras que la media de la escala con madres es inferior $(\mathrm{M}=4.54)$; respecto al placer en el Contacto físico con la CSP, las niñas y los niños evaluados tuvieron una $\mathrm{M}=$ 4.52 vs. $M=6.51$ con madres; en cuanto a la Interacción con otros adultos, la media de los infantes participantes fue mayor $(\mathrm{M}=6.11)$ a la media de la escala (5.08), por último, búsqueda Proximidad 
SENSIBILIDAD, COMPORTAMIENTO DE BASE SEGURA Y DESARROLlO Sociocognitivo en CEntros de educación Inicial en MÉxico

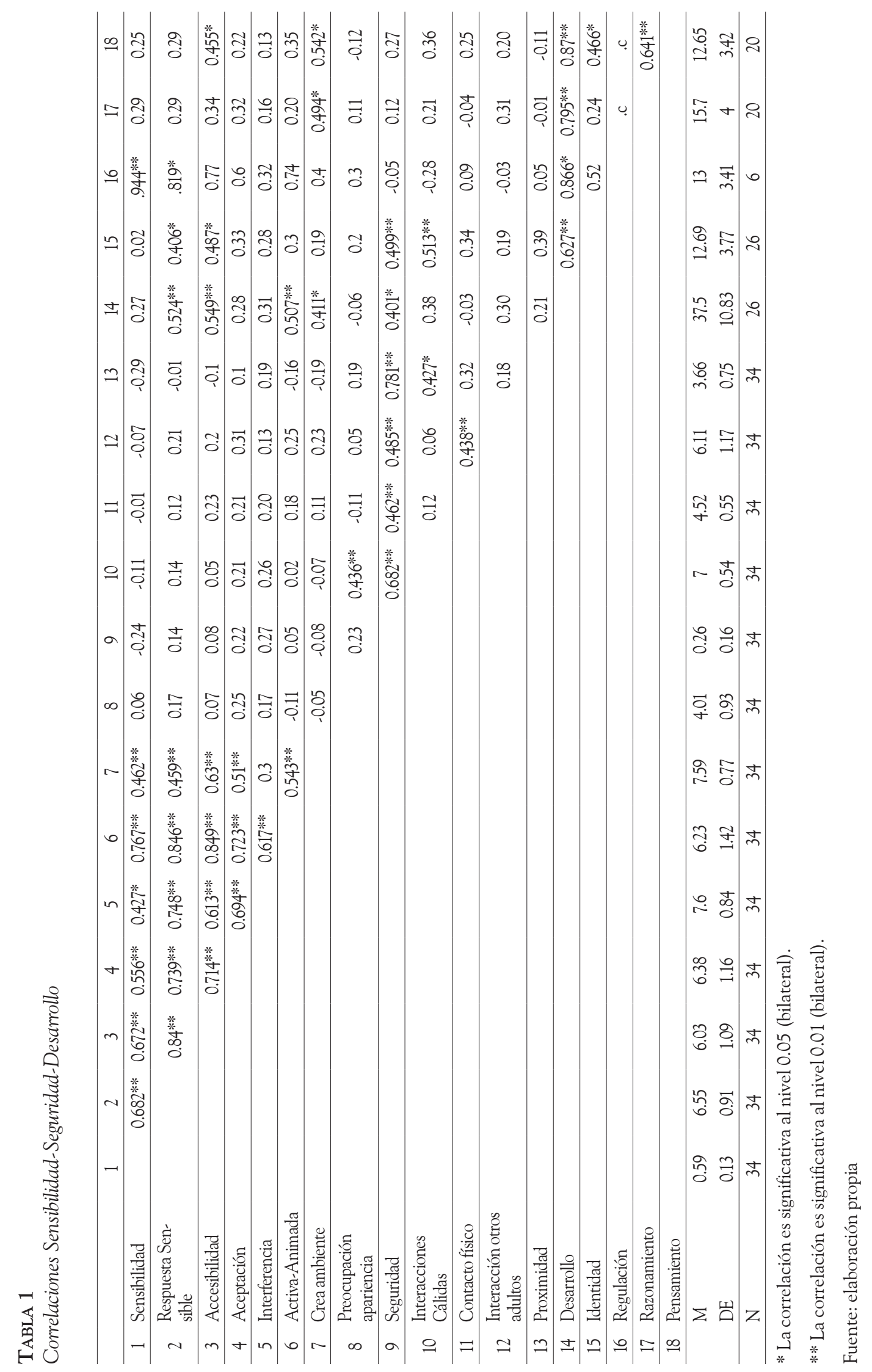

| Universitas Psychologica | V. 14 | No.3 | Julio-septiembre | 2015 | 
con la CSP tuvo una media de 3.66 en la muestra estudiada, inferior a la de infantes con sus madres $(\mathrm{M}=5.17)$.

En la Tabla 1 se, se presenta la matriz de correlación entre la sensibilidad de las CSP, el comportamiento de base segura y el desarrollo sociocognitivo de los infantes en CEI mediante análisis de correlación producto momento de Pearson.

\section{Discusión}

Las diferencias entre las medias de la sensibilidad de las CSP evaluadas y los promedios de las escalas indican que las tanto las CSP, como las madres: responden a las comunicaciones y señales de los infantes; son hábiles para tenerlos en cuenta a pesar de que compitan con otras demandas, como atender al resto del grupo; tienen un tono emocional predominantemente positivo al interactuar con los infantes y organizan ambientes estimulantes y apropiados, consecuencia de su experiencia y formación profesional. Empero, en ocasiones las CSP son incapaces de cooperar con ciertos comportamientos de los infantes y se encuentran ligeramente más interesadas que las madres en la presencia física adecuada de las niñas y los niños. Las diferencias entre dichos componentes de la sensibilidad resultan esperables, pues en los CEI existen demandas institucionales de cumplimiento con rutinas preestablecidas de acuerdo a reglas de operación diversas.

$\mathrm{El}$ que los infantes tuvieran menos de cuatro meses interactuando con la CSP $(M=3.69: D E=$ 1.76) resultó insuficiente para formar una relación de base segura, dicho de otra manera, construir un vínculo de apego. A diferencia del vínculo con la madre, en los CEI los infantes comparten y compiten por la atención de la cuidadora, lo cual hace que sus demandas no siempre sean atendidas de manera inmediata y que exista menor tiempo de interacción uno a uno, haciéndose más difícil atender a las necesidades particulares (Santelices \& Pérez, 2013). Estos hallazgos indican que los infantes están dispuestos a interactuar con sus CSP, así como a seguir sus sugerencias y órdenes, pero tienen menos contacto físico -y confort en el mismo- que el que sostienen niñas y niños de su edad con sus madres.
Asimismo, su experiencia en CEI hace que tengan una mayor disposición a interactuar y a disfrutar con distintos adultos; buscan menos cercanía con la CSP; están menos pendientes de su localización y su compañía no se vuelve imprescindible para realizar o iniciar actividades.

Si bien la presente investigación no contó con un grupo comparativo de infantes que no asisten a CEI, los puntajes obtenidos por las niñas y los niños participantes son congruentes a nivel teórico, ya que a través de su corta vida han experimentado diversidad de cuidados y estilos interactivos, lo que los hace ser más cautos en cuanto a la búsqueda de proximidad con las CSP, convirtiendo a la continuidad de la relación con sus madres en fuente primaria de seguridad para la exploración.

La sensibilidad de las CSP y el comportamiento de base segura de los infantes encontraron asociaciones estadísticamente significativas con su desarrollo sociocognitivo, por lo que se acepta la hipótesis de investigación. No fue posible evaluar el desarrollo sociocognitivo de los 34 infantes, sino solo 26, ya que, tal y como se explicó en el apartado de consideraciones éticas, si el infante estaba indispuesto o rechazaba participar, no se procedió con la evaluación. Otra variable que debe ser considerada, es el hecho de que existen dos versiones del instrumento: una para los infantes de un año (con dos categorías) y otra para los de dos años (tres categorías): Identidad personal y social es la única categoría presente en ambas versiones, por lo que, por ejemplo, Regulación, categoría de la versión de un año, contó únicamente con 6 respuestas.

Pese a lo anterior, el puntaje global de Seguridad de los infantes se asoció con su nivel total de Desarrollo $\left(r=0.401^{*}\right)$ en lo general y en lo particular, con su Identidad personal y social $(r=$ $0.499 * *)$, por lo que el uso habilidoso de la base segura en tiempos y contextos distintos, así como la certidumbre sobre la disponibilidad y responsividad del cuidador se relacionan con la confianza de los infantes en sus propias interacciones con el mundo, desarrollando en consecuencia autocontrol y reciprocidad, referentes sobre sí mismos y los otros (Juárez-Hernández et al., 2013; Weinfield, Sroufe, Egeland, \& Carlson, 1999). Acorde con 
SENSIBILIDAD, COMPORTAMIENTO DE BASE SEGURA Y DESARROLlO

sociocognitivo en Centros de educación inicial en MÉxico

esto, Interacciones cálidas también correlacionó significativamente con la Identidad personal $(r=$ $0.513 * *)$. Regulación se asoció con la Sensibilidad de la CSP $\left(r=0.944^{* *}\right)$. La respuesta de la CSP Activa-animada encontró vinculación con el nivel total de desarrollo $\left(r=0.507^{*}\right)$; su Respuesta sensible correlacionó con el Desarrollo de los infantes $\left(r=0.524^{* *}\right)$; con su Identidad personal y social $(r$ $\left.=0.406^{*}\right)$ y con su Regulación $\left(\mathrm{r}=0.819^{*}\right)$. Estos resultados, vistos como una gestalt, indican que la contestación oportuna y adecuada de la cuidadora a las comunicaciones y señales del infante se asocia con su capacidad para responder por su nombre; identificar parentescos; realizar por sí mismo actividades de exploración e higiénicas; seguir órdenes; relacionarse con compañeros y adultos y contenerse sin recurrir a berrinches/rabietas, así como al respeto a los límites corporales de una persona; a la regulación del comportamiento ante la negativa del adulto y a la expresión de agrado/desagrado y necesidades básicas. La correlación entre la respuesta sensible de la CSP y las capacidades cognitivas y sociales anteriormente descritas, puede explicarse por la influencia de CSP sensibles que se proponen a los infantes como base de seguridad y promueven el aprendizaje, resultado consistente con lo hallado en investigaciones afines (p. ej., Maldonado \& Carrillo, 2006; Peisner-Feinberg et al., 2001; Pianta \& Stuhlman, 2004).

La habilidad de la CSP para tener en cuenta al infante a pesar de que compita con otras demandas-Accesibilidad-correlacionó con Pensamiento deductivo emergente $\left(r=0.455^{*}\right)$, referido como el razonamiento infantil que hace alusión a las relaciones entre objetos concretos e ideas para resolver situaciones problemáticas de la vida diaria, esto gracias a la noción de conservación, el establecimiento de semejanza-diferencia, orden y causalidad. Se trata de un resultado interesante, pues el que precisamente la CSP tenga demandas varias, puede generar que los infantes en CEI resuelvan situaciones problemáticas distintas de las que tienen aquellos que no asisten a servicios de EI, por lo que se pone a prueba su capacidad de utilizar a los objetos y personas para resolver, entre otras cosas, la competencia con sus pares por la atención de la
CSP. Accesibilidad también encontró asociaciones con el puntaje global de Desarrollo $(r=0.549 * *)$ e Identidad personal y social $(r=0.487 *)$.

La organización que la CSP hace del entorno para convertirlo estimulante y apropiado para el infante, definida como Crea un ambiente interesante, encontró asociaciones estadísticamente significativas con el nivel total de Desarrollo $(r=$ $\left.0.411^{*}\right)$, con Razonamiento $(r=0.494)$ y con Pensamiento deductivo emergente $\left(r=0.542^{*}\right)$. Ya que el desarrollo es un proceso dinámico en continua transformación, producto de interacciones ambientales, la vinculación con CSP repercute en su curso.

Los CEI del IMSS tienen 2.5 más recursos económicos que lo que se asigna para la población no derechohabiente (Myers et al., 2013): es sabido que dentro de estos, los subrogados suelen tener mayores niveles de calidad, por lo que el correcto funcionamiento de los centros, sus instalaciones acondicionadas, la calidad de la interacción y los buenos resultados en el desarrollo sociocognitivo de los infantes participantes en este estudio no son, bajo ningún concepto, un reflejo de la realidad mexicana. Es necesario realizar investigación en CEI a los que asisten infantes sin acceso a seguridad social para poder cumplir con el objetivo propuesto por la Secretaría de Educación Pública (SEP) de ser un proceso con fines educativos que favorezca el desarrollo y aprendizaje de todos los niños y las niñas (SEP, 2012).

En concordancia con lo anterior, los CEI deben establecer rutinas de acuerdo a las necesidades y a los procesos de desarrollo de los infantes, ya que su estabilidad y predictibilidad favorecen la formación de vínculos de apego, la cooperatividad y la autorregulación vía la interacción CSP-infante (Santelices \& Pérez, 2013). Se recomienda que las autoridades de los CEI consideren la posibilidad de que la/s CSP permanezca/n con el mismo grupo de niñas y niños durante toda su estancia en el CEI -aproximadamente tres ciclos escolares de un año cada uno- para que aumenten las probabilidades de formar relaciones de base segura y que exista continuidad en los vínculos.

Si se toma en cuenta que las CSP no tienen lazos consanguíneos con los infantes y que se trata de una 
labor mal remunerada (percibiendo menos de 250 dólares mensuales), además de estar poco reconocida a nivel social (las madres y cultura popular las llaman "nanas" y ven su trabajo como "poca cosa"), su sensibilidad es extraordinaria. Santelices y Pérez (2013) subrayan el impacto del desgaste físico y emocional, asociados al ejercicio de la atención educativa a la primera infancia, y afirman que este repercute en el cumplimiento de sus objetivos y en la capacidad de responder a las demandas de los infantes. No solo se debe promover la formación continua del personal educativo, reforzar la relación familia-escuela y apoyar a las familias en sus prácticas de crianza (Farkas et al., 2015), sino que, antes de cumplir estas metas, es necesario crear mecanismos de selección de personal y promoción del mismo que privilegien factores no académicos, de manera que las CSP obtengan una remuneración más digna y sientan que su trabajo es valioso. CSP sensibles, sanas, motivadas y valoradas se encuentran en posibilidad de tomar cuantos cursos sea necesario para aumentar su nivel académico y conocimiento sobre desarrollo infantil temprano, por lo que se sugiere que los tomadores de decisiones amplíen su visión y consideren aspectos psicológicos e interpersonales/interactivos como precursores necesarios (Salinas-Quiroz, 2014).

Se recomienda que futuras investigaciones tomen en cuenta el nivel educativo y socioeconómico de las CSP y de las familias (p. ej., Santelices et al., 2015), así como las horas de práctica supervisada realizadas por las CSP (Farkas et al., 2015). Asimismo, podría contemplarse un grupo de comparación compuesto por infantes que no asisten a CEI y/o evaluar la sensibilidad de las madres y la relación de base segura madre-infante para explorar las similitudes y diferencias con la relación CSP-infante.

\section{Referencias}

Baker, J. A. (2006). Contributions of teacher-child relationships to positive school adjustment during elementary school. Journal of School Psychology, 44(3), 211-229.

Carbonell, O. A., Posada, G., Plata, S. J., \& Méndez, S. (2005). Las relaciones vinculares: un contexto para potenciar el derecho al bienestar de niños y niñas. Cuadernos de Psicología, 1, 31-38.

Cárcamo, R. A., Vermeer, H. J., De la Harpe, C., van der Veer, R., \& van Ijzendoorn, M. H. (junio, 2014). The quality of childcare in Chile: Its stability and international ranking. Child Youth Care Forum, 1-15. doi: 10.1007/s10566-014-9264-z

Crittenden, P. (2005). Care-Index para toddlers: Manual de Codificación. Miami, EEUU: Family Relations Institute.

Farkas, C., Carvacho, C., Galleguillos, F., Montoya, F., León, F., Santelices, M. P., \& Himmel, E. (2015). Estudio comparativo de la sensibilidad entre madres y personal educativo en interacción con niños y niñas de un año de edad. Perfiles Educativos, 37(148), 16-33.

Fondo Internacional de Emergencia de las Naciones Unidas para la Infancia. (1989). Convención sobre los Derechos del Niño. Nueva York: Autor.

Friedman, S., \& Boyle, D. E. (2008). Attachment in US children experiencing nonmaternal care in the early 1990s. Attachment and Human Development, 10(3), 225-261.

Gunnar, M. R., Tagle, N. M., \& Herrera, A. (2009). Stressor paradigms in developmental studies: What does and does not work to produce mean increases in salivary cortisol. Psychoneuroendocrinology, 34(7), 953-967. doi: 10.1016/j. psyneuen.2009.02.010

Howes, C., \& Ritchie, S. (2002). A matter of trust: Connecting teachers and learners in the early childhood classroom. New York: Teachers College.

Juárez-Hernández, M. C. (2004a). Traducción al castellano del MBQS. Manuscrito inédito, Área Académica Aprendizaje y Enseñanza en Ciencias Humanidades y Artes, Universidad Pedagógica Nacional, Ajusco, México.

Juárez-Hernández, M. C. (2004b). Traducción al castellano del AQS. Manuscrito inédito, Área Académica Aprendizaje y Enseñanza en Ciencias Humanidades y Artes, Universidad Pedagógica Nacional, Ajusco, México.

Juárez-Hernández, M. C., del Valle, L., Delgado, A. O., Pérez, G., Tlalpachícatl, N., \& de Castro, F. (2013). Validación de dos escalas de capacidades cognitivas y sociales para niños de uno y dos años. Manuscrito 
SENSIBILIDAD, COMPORTAMIENTO DE BASE SEGURA Y DESARROLLO

SOCIOCOGNITIVO EN CENTROS DE EDUCACIÓN INICIAL EN MÉXICO

inédito, Área Académica Aprendizaje y Enseñanza en Ciencias Humanidades y Artes, Universidad Pedagógica Nacional, Ajusco, México.

Kienbaum, J. (2001). The socialization of compassionate behavior by child care teachers. Early Education $\mathbb{8}$ Development, 12(1), 139-153.

Magnuson, K. A., Ryhm, C. J., \& Waldfogel, J. (2004). Does prekindergarten improve school preparation and performance? Cambridge, MA: National Bureau of Economic Research.

Maldonado, C., \& Carrillo, S. (2006). Teaching with affection: Characteristics and determinant factors of quality in teacher student relationships. Journal Childhood Adolescent and Family, 1(1), 39-60.

Mashburn, A. J., Pianta, R. C., Hamre, B. K., \& Downer, J. T. (2008). Measures of classroom quality in prekindergarten and children's development of academic, language, and social skills. Child Development, 79(2), 732-749.

México. Ley General de Educación. 28 de abril de 2011. DOF 28-01-2011.

México. Ley General de Prestación de Servicios para la Atención, Cuidado y Desarrollo Integral Infantil. DOF 24-10-2011.

Myers, R., Martínez, A., Delgado, M. A., Fernández, J. L., \& Martínez, A. (2013). Diagnóstico infantil temprano en México. Diagnóstico y recomendaciones (Resumen Ejecutivo). Washington DC: Banco Interamericano de Desarrollo. División de Protección Social y Salud.

Oren, M. (2006). Child temperament, gender, teacher-child relationship, and teacher-child interactions (Tesis doctoral inédita, Universidad Estatal de Florida, EE. UU.). Recuperado de http://diginole.lib.fsu.edu/ cgi $/$ viewcontent.cgi? article $=3890 \&$ context $=$ etd

Organización de las Naciones Unidas para la Educación, la Ciencia y la Cultura. (2012). EFA global monitoring report, 2012: Expanding equitable early childhood care and Education is an urgent need. Paris: Autor. Recuperado de http://unesdoc.unesco.org/ images/0021/002160/216038E.pdf

Pederson, D. R., \& Moran, G. (1995). Appendix B. Maternal Behavior Q-set. En E. Waters, B. E. Vaughn, G. Posada \& K. Kondo-Ikemura (Eds.), Caregiving, cultural, and cognitive perspectives on secure-base behavior and working models: New growing points of attachment theory and research. Monographs of the Society for Research in Child Development, $60(2-3), 247-254$.

Peisner-Feinberg, E., Burchinal, M., Clifford, R., Culkin, M., Howes, C., Lynn Kagan, S.L., \& Yazejian, N. (2001). The relation of preschool child-care quality to children's cognitive and social developmental trajectories through second grade. Child Development, 72(5), 1534-1553.

Pianta, R. C., \& Stuhlman, M. (2004). Teacher-child relationships and children's success in the first years of school. Social Psychology Review, 33(3), 444-458.

Posada, G., Jacobs, A., Carbonell, O. A., Alzate, G., Bustamante, M. R., \& Arenas, A. (1999). Maternal care and attachment security in ordinary and emergency context. Developmental Psychology, 35(6), 1379-1388.

Posada, G., Jacobs, A., Richmond, M., Carbonell, O. A., Alzate, G., Bustamante, M. R., \& Quiceno, J. (2002). Maternal caregiving and infant security in two cultures. Developmental Psychology, 38(1), 67-78.

Posada, G., Waters, E., Crowell, J., \& Lay, K. (1995). Is it easier to use secure mother as a secure base? Attachment Q-sort correlates of the Adult Attachment Interview. En E. Waters, B. Vaughn, G. Posada \& K. Kondo-Ikemura (Eds.), Caregiving, cultural and cognitive perspectives on securebase behavior and working models. New Growing Points of Attachment Theory and Research. Monographs of the Society for Research in Child Development, 60(2-3, Serie 244), 133-178.

Roisman, G. I., \& Fraley, R. C. (2008). A behaviorgenetic study of parenting quality, infant attachment security, and their covariation in a nationally representative sample. Developmental Psychology, 44(3), 831-839.

Salinas-Quiroz, F. (2014). Educación Inicial de Base Segura: Estudio observacional de los procesos internos de la educadora, de la interacción y del desarrollo sociocognitivo infantil (Tesis de Doctorado inédita, Universidad Nacional Autónoma de México, México).

Salinas-Quiroz, F. (2013). Vínculos de apego con cuidadores múltiples: la importancia de las relaciones afectivas en la Educación Inicial [CD-ROM]. Memorias Electrónicas del XII Congreso Nacio- 
nal de Investigación Educativa. México: COMEI. doi: 10.13140/2.1.1043.8083

Salinas-Quiroz, F., Morales-Carmona, F., Cruz-Martínez, L. M., Posada, G., \& Carbonell, O. A. (2014). Aproximación psicométrica al uso de los Q-Sorts en contextos de Educación Inicial. Acta de Investigación Psicológica, 4(2), 1595-1611.

Salinas-Quiroz, F. \& Posada, G. (2015). MBQS: Método de evaluación para intervenciones en apego dirigidas a primera infancia. Revista Latinoamericana de Ciencias Sociales, Niñez y Juventud, 13(2), 1051-1063.

Salinas-Quiroz, F., Morales-Carmona, F., de Castro, F., Juárez-Hernández, M.C., Posada, G. \& Carbonell, O. A. (2015). Educación Inicial de Base Segura: Indicador de la calidad educativa para la primera infancia. Psicología Iberoamericana, 23(1), 75-82.

Santelices, M. P., \& Pérez, F. (2013). Evolución del vínculo que establece el niño con el personal educativo en salas cuna chilenas. Universitas Psychologica, 12(2), 821-832. doi:10.11144/Javeriana. UPSY12-3.even

Santelices, M. P., Carvacho, C., Farkas, C., León, F., Galleguillos, F., \& Himmel, E. (2012). Medición de la sensibilidad del adulto con niños de 6 a 36 meses de edad: construcción y análisis preliminares de la Escala de Sensibilidad del Adulto, E.S.A. Terapia Psicológica, 30(3), 19-29.

Santelices, M. P., Farkas, C., Montoya, M. F., Galleguillos, F., Carvacho, C., Fernández, A., ... Himmel, E. (2015). Factores predictivos de sensibilidad materna en infancia temprana. Psicoperspectivas, 14(1), 66-76. doi:10.5027/PSICOPERSPECTIVASVOL14- ISSUE1-FULLTEXT-441

Santelices, M. P., Greve, G. C., \& Pereira, X. (2015). Relación entre la interacción del preescolar con el personal educativo y su desarrollo psicomotor: un estudio longitudinal chileno. Universitas Psychologica, 14(1), 313-328. doi: 10.11144/Javeriana. upsy14-1.ripp
Secretaría de Educación Pública. (2012). Modelo de atención con enfoque integral. México: Autor.

Silver, R. B., Measelle, J. R., Armstrong, J. M., \& Essex, M. J. (2005). Trajectories of classroom externalizing behavior: Contributions of child characteristics, family characteristics, and the teacher-child relationship during the school transition. Journal of School Psychology, 43(1), 39-60.

Sociedad Mexicana de Psicología. (2007). Código ético del psicólogo: normas de conducta, resultados de trabajo, relaciones establecidas. México: Trillas.

Universidad Pedagógica Nacional. (2011). Escala de Evaluación de Calidad de Centros de Educación Inicial (Vol. 4.0). Manuscrito inédito.

Vandell, D., Belsky, J., Burchinal, M., Steinberg, L., Vandergrift, N., \& the NICHD Early Child Care Research Network. (2010). Do effects of early child care extend to age 15 years? Results from the NICHD Study of Early Child Care and Youth Development. Child Development, 81(3), 737-756. doi:10.1111/j.1467- 8624.2010.01431.x

Vermeer, H. J., \& Bakermans-Kranenburg, M. J. (2008). Attachment to mother and nonmaternal care: Bridging the gap. Attachment $\mathcal{E}$ Human Development, 10(3), 263-273.

Waters, E. (1995). Attachment Q-set items. En E. Waters, B. Vaughn, G. Posada \& K. Kondo-Ikemura (Eds.), Caregiving, cultural and cognitive perspectives on secure-base behavior and working models: New growing points of attachment theory and research. Monographs of the Society for Research in Child Development, 60(2-3; Serial 209), 255-265. doi:10.1111/j.1540-5834.1995.tb00214.x

Weinfield, N. S., Sroufe, L. A., Egeland, B., \& Carlson, E. A. (1999). The nature of individual differences in infant-caregiver attachment. En J. Cassidy \& P. Shaver (Eds.), Handbook of attachment: Theory, research and clinical applications (pp. 68-88). New York: The Guilford Press. 